\title{
Does Nasal Obstruction Increase Heart Rate?
}

\author{
Venkatesha Belur Keshavamurthy, ${ }^{1}$ Munish Kambathatti Shekharappa, ${ }^{1}$ Yogeesha Beesanahalli, ${ }^{1}$ Nagaraj Maradi, ${ }^{1}$ Priya \\ Rani Kori ${ }^{1}$
}

\begin{abstract}
$\underline{\text { Introduction }}$
Nasal obstruction is implicated in the etiopathogenesis of Obstructive Sleep Apnea (OSA). OSA is associated with mean heart rate (HR) variations in wakefulness and in sleep. Early intervention has proven to reduce cardiovascular morbidity in OSA patients. In spite of various confounding factors HR measurement has been utilised as an independent predictor of mortality. The influence of severity of nasal obstruction on HR has not been studied in the literature. This study aims to clarify the influence of severity of nasal obstruction on HR.

Materials and Methods

We examined 55 patients aged less than 50 years with no previous cardiac complaints, who underwent overnight oxygen saturation and HR monitoring. The patients were divided into Mild, Moderate and Severe Nasal Obstruction group depending on NOSE scale grading.

$\underline{\text { Results }}$

There was no statistically significant difference in the Mean HR, Min HR, Max HR, and Max-Min HR in mild, moderate or severe nasal obstruction groups.

$\underline{\text { Discussion }}$

The role of nasal obstruction in Obstructive Sleep Apnoea and the importance of HR as predictor of cardiovascular morbidity have been discussed. The studies on the heart rate in nasal obstruction and OSA were reviewed.

Conclusion

Nasal obstruction does not influence the heart rate.

Keywords: Heart Rate, Obstructive Sleep Apnea, Nasal Obstruction
\end{abstract}

ABSTRACT

$\mathrm{N}$ asal obstruction is implicated in the etiopathogenesis of Obstructive sleep apnea (OSA). OSA is associated with mean heart rate (HR) variations in wakefulness and in sleep. ${ }^{1}$ Nasal obstruction is caused by various pathologies like deviated nasal septum, hypertrophied turbinates, vasomotor phenomenon, sinonasal polyps, sinusitis, allergy and chronic granulomatous nasal conditions.

Nasal obstruction compels mouth breathing and during sleep, due to greater negative pressure which develops in the oropharynx area, pliable structures like soft palate, uvula, vibrate leading to snoring. The Starling resistor

\section{1 - S.S. Institute of Medical Sciences \& Research Centre, Davangere, Karnataka}

\section{Corresponding author:}

Dr Venkatesha Belur Keshavamurthy

email: bk.venkatesha@gmail.com model of upper airway collapsibility describes how the upper airway behaves like a Starling resistor, in that obstruction at the inlet (i.e. the nasal airway) produces collapsing forces, that manifest downstream in the collapsible segment, the pharynx. ${ }^{2}$

Nasal obstruction results in nocturnal mouth breathing, sleep fragmentation ${ }^{3}$ and snoring, leading to sequele of disturbed sleep ${ }^{4,5}$ like excessive daytime sleepiness. Definite role of nasal obstruction in complex etiopathogenesis of Obstructive sleep apnea (OSA) has been proven in the literature. ${ }^{4,6}$

Association between snoring / OSA and adverse cardiovascular event is well documented. Time-domain analysis of heart rate (HR) variability, used as the only criterion, could represent an efficient tool in OSAS diagnosis with a sensitivity of $90 \% .{ }^{7}$ Heart rate variability is an important parameter measured for the assessment of 
autonomic nervous system activity indirectly measuring the adverse cardiovascular outcome. Furthermore elevated Mean HR was reported to be a risk factor for cardiovascular mortality in normotensive patients with end stage renal disease. ${ }^{8} \mathrm{HR}$ represents one of the most important independent predictors of cardiovascular, noncardiovascular, and overall mortality. ${ }^{9}$

HR can easily be monitored in various clinical setting. Few literature reviews are available implicating experimentally induced nasal obstruction as an independent factor involved in increase of HR. ${ }^{10}$ But role of spontaneous nasal obstruction as an independent factor in increasing the HR has not been proved in the literature. This study is intended to analyze the relationship of severity of nasal obstruction to mean $\mathrm{HR}$, thus indirectly predicting adverse cardiac as well as overall mortality.

\section{Materials and Methods}

A cross-sectional study involving 55 patients, who underwent septoplasty and or turbinate reduction between November 2014 to April 2016 for various indications like nasal obstruction, Sluders neuralgia, epistaxis, and as an approach to DCR and FESS. Subjects with known cardiovascular diseases, on medications with beta blockers, hyperthyroidism and OSA (Epworth Sleepiness Scale score $>8$ ) were excluded from the

Table I: Patient Characteristics

\begin{tabular}{|c|c|c|c|c|c|}
\hline PATIENT & $\begin{array}{l}\text { "GROUP I } \\
(\mathbf{N}=\mathbf{1 0}) "\end{array}$ & $\begin{array}{l}\text { "GROUP II } \\
(\mathbf{N}=\mathbf{2 5}) "\end{array}$ & $\begin{array}{l}\text { "GROUP III } \\
(\mathbf{N}=11) "\end{array}$ & $\begin{array}{l}\text { "GROUP IV } \\
(\mathrm{N}=09) "\end{array}$ & P VALUE \\
\hline Age & $\begin{array}{l}\text { "16-28yrs } \\
\text { Mean } 20.2 \\
\text { yrs" }\end{array}$ & $\begin{array}{c}\text { "16-45 yrs } \\
\text { Mean 28.2 yrs" }\end{array}$ & $\begin{array}{c}\text { "16-44 yrs } \\
\text { Mean 27.64 } \\
\text { yrs" }\end{array}$ & $\begin{array}{c}\text { "24-50yrs } \\
\text { Mean=36.78 } \\
\text { yrs" }\end{array}$ & $\begin{array}{l}" F=5.811 \\
p=0.0017 "\end{array}$ \\
\hline Male Gender & $9(90 \%)$ & $17(68 \%)$ & $10(90.9 \%)$ & $6(66.67 \%)$ & $\begin{array}{c}\text { "F=1.247 } \\
p=0.30 "\end{array}$ \\
\hline $\begin{array}{c}\text { Septal Deviation } \\
\text { with concomitant } \\
\text { Turbinate } \\
\text { pathology }\end{array}$ & $1(10 \%)$ & $3(12 \%)$ & $3(27.3 \%)$ & $2(22.2 \%)$ & $\begin{aligned} " F & =0.5838 \\
p & =0.63 "\end{aligned}$ \\
\hline
\end{tabular}

study. Subjects were given NOSE (Nasal obstruction and septoplasty evaluation) scale ${ }^{11}$ and depending on the subjective grading of the severity of the obstruction, they were divided into 4 groups: No obstruction group, mild obstruction group, moderate obstruction group and severe obstruction group.

On the preoperative day, overnight monitoring heart rate was done for minimum of 6 hours. Clinical parameters like Mean Heart Rate (MHR) Minimum Heart Rate (Min HR) Maximum Heart rate (Max HR) and Difference of Maximum and Minimum Heart rate (Max-Min HR) were recorded using 24 hour pulseoximetry.

Statistical tests: Continuous data were expressed as mean and SD. Spearman's Correlation coefficient test was used to determine the relationship between degree of nasal obstruction and Mean HR, Max HR, Min HR, Max-Min HR, Intergroup variability and significance was determined by one way ANOVA test. Linear regression analysis was performed to determine the relation of severity of nasal obstruction to $H R$. A p value of $<0.05$ was considered statistically significant.

\section{Results}

Clinical characteristics of the patients are shown as in Table I. Group IV had higher age group distribution compared to the similar age distribution in other groups. 
All groups had predominant Male gender population and septal deviation was the predominant nasal pathology in all groups.

Overnight Heart Rate measurement revealed Mean HR of 74.6, 75.24, 74.91 and 71.78, Max HR was 105.6, 103.28, 106.54 and 102.33; Max-Min HR was $44.2,42.64,45.54,43.89$ respectively in Group I-IV as shown in Table II.
The association between the two variables would not be considered statistically significant and are negatively weakly correlated. For HR fluctuation (Max-Min HR) the value of correlation coefficient value was 0.02839 and the two-tailed value of $\mathrm{P}$ was 0.837 indicating very weak positive relationship for Max-Min HR. These results clearly indicate there is no statistically significant relationship between the severity of Nasal Obstruction

Table II: Overnight Heart Rate monitoring findings

\begin{tabular}{|c|c|c|c|c|c|}
\hline $\begin{array}{l}\text { HEART RATE } \\
\text { PARAMETER }\end{array}$ & $\begin{array}{l}\text { "GROUP I } \\
(\mathbf{N}=\mathbf{1 0}) "\end{array}$ & $\begin{array}{l}\text { "GROUP II } \\
(\mathbf{N}=25) "\end{array}$ & $\begin{array}{l}\text { "GROUP III } \\
(\mathrm{N}=11) "\end{array}$ & $\begin{array}{l}\text { "GROUP IV } \\
(\mathbf{N}=09) "\end{array}$ & P VALUE \\
\hline Mean HR & $\begin{array}{c}" 74.6 \\
(70-78) "\end{array}$ & $\begin{array}{c}" 75.24 \\
(67-80) "\end{array}$ & $\begin{array}{c}" 74.91 \\
(70-80) "\end{array}$ & $\begin{array}{c}" 71.78 \\
(57-80) "\end{array}$ & $\begin{array}{c}" F=1.759 \\
p=0.17 "\end{array}$ \\
\hline Max HR & $\begin{array}{c}" 105.6 \\
(100-110) "\end{array}$ & $\begin{array}{l}" 103.28 \\
(96-114) "\end{array}$ & $\begin{array}{c}" 106.54 \\
(90-115) "\end{array}$ & $\begin{array}{c}" 102.33 \\
(96-112) "\end{array}$ & $\begin{array}{c}" F=1.253 \\
p=0.30 "\end{array}$ \\
\hline Min HR & $\begin{array}{c}" 61.6 \\
(58-66) "\end{array}$ & $\begin{array}{c}" 60.64 \\
(56-66) "\end{array}$ & $\begin{array}{c}" 61 \\
(56-66) "\end{array}$ & $\begin{array}{l}" 57.33 \\
(49-68) "\end{array}$ & $\begin{array}{l}" F=2.831 \\
p=0.047 "\end{array}$ \\
\hline Max-Min HR & $\begin{array}{c}" 44.2 \\
(40-50) "\end{array}$ & $\begin{array}{c}" 42.64 \\
(36-54) "\end{array}$ & $\begin{array}{c}" 45.54 \\
(34-61) "\end{array}$ & $\begin{array}{c}\text { "43.89 } \\
(36-53) "\end{array}$ & $\begin{aligned} " F & =0.6906 \\
p & =0.56 "\end{aligned}$ \\
\hline
\end{tabular}

Spearman's correlation coefficient value was -0.15 and the two tailed $\mathrm{p}$ value was 0.27583 , which indicates there is very weak negative monotonic relationship between Mean HR and severity of the nasal obstruction and as $p$ value is more than 0.05 , results were statistically insignificant.

Similarly, Spearman's correlation test was done to find the relationship between Max HR, Min HR, MaxMin HR and Min HR. For Max HR the correlation coefficient value was -0.15316 and the two-tailed value of $\mathrm{P}$ were 0.26427 indicating very weak negative relationship without statistical significance. For Min $\mathrm{HR}$, The value of correlation coefficient was -0.25683 and the two-tailed value of $\mathrm{P}$ is 0.05838 . and HR parameters.

A simplelinear regression was calculated to predictHR based on severity of nasal obstruction. A Non significant regression equation was found, $\mathrm{R} 2=1.901752424 .10-1$, $\mathrm{Y}=3.138205319 .10-2 \quad, \mathrm{X}=2.246768581$, considering participants' predicted increased HR was equal to or more than 80.8 When severity of nasal obstruction was measured as no obstruction, mild, moderate, severe nasal obstruction, HR did not increase for each degree of nasal obstruction. A scatter plot diagram shows the linear regression as shown in Fig.1. An Analysis of Variance showed that the effect of degree of nasal obstruction was not significant $\mathrm{F}=1.756, \mathrm{p}=0.167$. 


\section{Discussion}

Role of Nasal obstruction in OSA: Nasal obstruction is caused by various pathologies like deviated nasal septum, hypertrophied turbinates, vasomotor phenomenon, sinonasal polyps, sinusitis, allergy and chronic granulomatous nasal conditions. Significant role of nasal obstruction in the etiopathogenesis of OSA is debatable. Mc Nicholas describes the starling resistor model for the development of OSA. Nose being the inlet of the respiratory tube, obstruction of the inlet snoring in a high percentage of cases. Numerous studies examining the effects of experimentally induced nasal obstruction (ie nasal packing) on OSA have indicated a positive association. In predisposed individuals, treatment of allergy has proven a vital role in reducing obstructive sleep apnea. ${ }^{6}$

Even though NO plays a minor role in the complex pathogenesis of OSA, but has relevance in the aetiology of snoring. ${ }^{12}$ The switchover from nasal breathing to mouth breathing is crucial in determining the pathophysiology of OSA. ${ }^{2}$ Nasal and oral resistance,

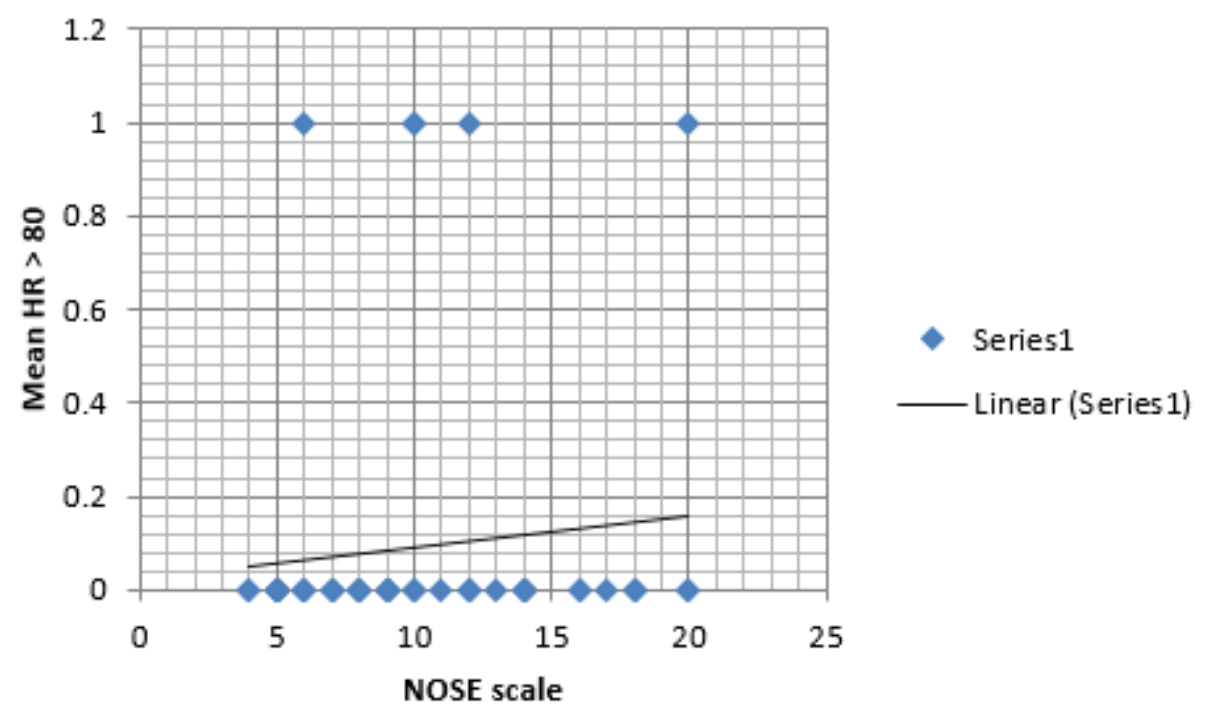

Fig. 1. Showing scattered plot depicting the relationship between increased HR and nasal obstruction.

leading to the development of negative pressure in the oropharynx leading to collapse of compliant pharyngeal structures. ${ }^{2}$ It was observed that fixed nasal obstruction as seen in deviated nasal septum, polyps, hypertrophied turbinates, contributes less when compared to variable nasal obstruction like reversible nasal congestion and allergy. ${ }^{2}$ Since breathing through the nose appears to be the preferred route during sleep, nasal obstruction frequently leads to nocturnal mouth breathing, snoring, and ultimately to OSA.

It has also been demonstrated that nasal stimulation or obstruction determines an increase in the lung airways resistance. In normal subjects, nasal obstruction partial or total, due to various causes (septal deviation, turbinate hypertrophy and other nasal abnormalities) provokes tone of the muscles, architecture of the upper airway, posture of the individual, age of the individual, obesity and medications influences OSA.

Clearing nasal obstruction relieves snoring in few, but some continue to snore indicating multifactorial nature of OSA. ${ }^{2,12}$

Use of external nasal devices, ${ }^{13}$ surgery for reducing the nasal obstruction like turbinoplasties, correction of nasal septum, removing the polyps have successfully reduced nasal resistance and severity of snoring, but failed to improve AHI, RDI, Arousal Index, total sleep time, sleep architecture, sleep fragmentation, including snoring loudness. ${ }^{3}$

Furthermore, surgical intervention for OSA was 
clearly successful in children and adults where a long segment of upper airway is corrected like removing tonsils, reducing the tongue base, osteotomies and combination procedures, than short segment surgery like isolated correction of soft palate UPPP, septoplasty, isolated turbinate correction which has met with more failure rates. ${ }^{4}$

HR as predictor of Cardiovascular Morbidity: It's a general agreement that heart rate represents one of the most important independent predictor of cardiovascular, non-cardiovascular and overall mortality. ${ }^{9}$ Mean HR was reported to be a risk factor for cardiovascular mortality. ${ }^{1}$ In a study by Fulvia Seccareccia, involving 2533 men aged between 40-69 with other variable, calculated the risk of mortality against increase in the HR. ${ }^{9}$

It was found when other risk factors being equal, death risks increase about $50 \%$ for each 20 - beatper-minute increment, and relative risks between extreme HR levels are more than 2-fold. ${ }^{9}$ Heart rate exhibits great inter individual variability: is influenced by stress, anxiety, ${ }^{9}$ physical activity, psychological makeup, hormonal status, gender etc. It also shows intra individual variation, influenced by circadian rhythm, stages of sleep. Resting heart rate i.e. HR measured during sleep is of more clinical importance than heart rate measured during wakefulness.

Factors like nature of the surgery, less effective pain control, sleep deprivation following surgery, type of nasal packing influences the basal heart rate measurements in the post operative period. Hence preoperative measurement of HR gives near physiological values than post operative measurement. HR monitoring can be easily done with the use of pulse oximetry in resource poor setting.

The differences between healthy and OSA subjects were much smaller between sleep stages indicating similar mechanism for heart rate control in both healthy and OSA patients. ${ }^{14}$ The severity of OSA was independently associated with elevated mean HRs during 24 hours, wakefulness, and sleep in patients with OSA and six month treatment with nCPAP reduced the values in those patients. The prognostic significance of elevated mean HRs during 24 hours, wakefulness and sleep is necessary to be clarified in patients with OSA.
Le Heuzey et al described greater visual enlargement of the RR trend and a significantly higher difference between minimal and maximal HR during the night in OSAS patients than in control subjects. ${ }^{7}$ In our current study, higher difference between minimal and maximal HR during night monitoring did not signify any correlation with severity of nasal obstruction.

In a pilot study by John Hart, it was observed that a higher Resting Pulse Rate (RPR) at baseline was associated with increased RPR change, whereas a lower baseline RPR was associated with a stable or reduced RPR change. ${ }^{15}$ In a Study by Michael S. Benninger, on athletes to detect the effect of nasal obstruction, on workload, respiratory rate, Mean Heart rate, oxygen saturation, systolic BP, did not find any statistically significant difference in these parameters. Our results matches with this study, with reference to the Mean heart rate. ${ }^{10}$

\section{Conclusion}

Mean heart rate, Maximum Heart Rate and difference between Max and min HR is not affected by severity of nasal obstruction.

Limitations of the study: Comparison between two objective values (like rhinomanometry, flow, resistance values) may provide a better correlation than assessing subjective scores (NOSE score) with HR. Measurement of HR with reference to various stages of sleep would indicate the effect of nasal obstruction on autonomic control mechanisms of CVS. Concomitant measurement of ECG, with various time domain parameters of HR variability would have given more predictability in terms of adverse cardiovascular events.

\section{References}

1. Kawano Y, Tamura A, Wantabe T, Kadota J, Influence of the severity of obstructive sleep apnea on heart rate Journal of Cardiology:2010: 56; 27-34

2. Mc Nicholas, The Nose and OSA: Variable nasal obstruction may be more important in pathophysiology than fixed obstruction. Eur Respir J. 2008; 32: 3-8

3. Migueisa D.P, Thuler L.C.S, Lemesc NA, Moreira C.S.S, Joffily L, Araujo-Melo MH. Systematic review: the influence of nasal obstruction on sleep apnea: Braz J Otorhinolaryngol. 


\section{6; 82(2):223-31.}

4. Scharf MB, Cohen AP. Diagnostic and treatment implications of nasal obstruction in snoring and obstructive sleep apnea. Ann Allergy Asthma Immunol. 1998 Oct; 81(4):279-87.

5. Prota R, Dalmasso F. Snoring: analysis, measurement, clinical implications and application. Eur Respir J. 1996, 9: 146-59.

6. Pevernagie DA, De Meyer MM, Claeys S. Sleep, breathing and the nose: Sleep Medicine Reviews 2005: 9: 437-51.

7. Roche R, Gaspoz JM, Court-Fortune I, Minini P, Pichot V, Duverney D, et al. Screening of Obstructive Sleep Apnea Syndrome by Heart Rate Variability Analysis: Circulation 1999: 100:1411-5.

8. Cice G, Di Benedetto A, Isa S, De Gregorio P, Marcelli D, Gatti E, Calabrò R.. Heart rate as independent prognostic factor for mortality in normotensive hemodialysed patients. J Nephrol. 2008; 21(5):704-12.

9. Seccareccia F, Pannozzo F, Dima F, Minoprio A, Mendittlo A, Noce CL, et al. Heart Rate as a Predictor of Mortality: The MATISS Project: American Journal of Public Health 2001: 91(8): 1258-63.
10. Benninger MS, Sarpa JR, Ansari Tariq, Ward J. Nasal patency, aerobic capacity, and athletic performance. OtolaryngologyHead and Neck Surgery 1992:107(2):101-4

11. Stewart MG, Witsell DL, Smith TL, Weaver EM, Yueh B, Hannley MT. Development and validation of the Nasal Obstruction Symptom Evaluation (NOSE) scale. Otolaryngol Head Neck Surg. 2004;130(2):157-63

12. Kohler M, Bloch K.E, Stradling JR. The role of nose in the pathogenesis of obstructive sleep apnea and snoring. Eur Respir J. 2007; 30: 1208-15

13. Pevernagie, D, Hamans E, Cauwenberge V, Pauwels R. External nasal dilation reduces snoring in chronic rhinitis patients: a randomized controlled trial. Eur Respir J. 2000; 15: 996-1000

14. Penzel T, Kantelhardt JW, Lo CC, Voigt K, Vogelmeier C. Dynamics of Heart Rate and Sleep Stages in Normals and Patients with Sleep Apnea. Neuropsychopharmacology 2003; 28;48-53

15. Hart J, Testing an association between baseline resting pulse rate averages and short-term changes in resting pulse rates: A pilot study J Can Chiropr Assoc. 2015; 59(2):165-72. 